\title{
Identification of mRNAs related to endometrium function regulated by IncRNA CD36-005 in rat endometrial stromal cells
}

\author{
Xueying Zhang ${ }^{1}$, Ying Xu' ${ }^{1}$, Lulu Fu', Dandan Li', Xiaowei Dai', Lianlian Liu', Jingshun Zhang ${ }^{1}$, \\ Lianwen Zheng $^{1 *}$ and Manhua Cui ${ }^{2^{*}}$
}

\begin{abstract}
Background: Polycystic ovary syndrome (PCOS) is a heterogeneous endocrine disorder in women of reproductive age and is commonly complicated by adverse endometrial outcomes. Long non-coding RNAs (IncRNAs) are a class of non-protein-coding transcripts that are more than 200 nucleotides in length. Accumulating evidence indicates that IncRNAs are involved in the development of various human diseases. Among these IncRNAs, IncRNA CD36-005 (CD36-005) is indicated to be associated with the pathogenesis of PCOS. However, the mechanisms of action of CD36-005 have not yet been elucidated.
\end{abstract}

Methods: This study determined the CD36-005 expression level in the uteri of PCOS rat model and its effect on the proliferation activity of rat primary endometrial stromal cells. RNA sequencing (RNA-seq) and bioinformatics analysis were performed to detect the mRNA expression profiles and the biological pathways in which these differentially expressed mRNAs involved, after CD36-005 overexpression in the primary endometrial stromal cells. The differential expression of Hmgn5, Nr5a2, DII4, Entpd1, Fam50a, and Brms1 were further validated by quantitative reverse transcription polymerase chain reaction (qRT-PCR).

Results: CD36-005 is highly expressed in the uteri of PCOS rat model and promotes the proliferation of rat primary endometrial stromal cells. A total of fifty-five mRNAs differentially expressed were identified in CD36-005 overexpressed stromal cells. Further analyses identified that these differentially expressed mRNAs participate in many biological processes and are associated with various human diseases. The results of qRT-PCR validation were consistent with the RNA-seq data.

Conclusions: These data provide a list of potential target mRNA genes of CD36-005 in endometrial stromal cells and laid a foundation for further studies on the molecular function and mechanism of CD36-005 in the endometrium.

Keywords: IncRNA, CD36-005, RNA sequencing, Endometrium, Stromal cells, PCOS

\section{Background}

Polycystic ovary syndrome (PCOS) is one of the most common and complex endocrine disorders in women of reproductive age, with a prevalence estimated to be $5-10 \%$ [1-4]. The clinical features of PCOS are highly heterogeneous. Patients with PCOS have reproductive dysfunction and metabolic abnormalities, and are

\footnotetext{
*Correspondence: davezheng@sohu.com; jlucmh@163.com

${ }^{1}$ Reproductive Medical Center, Department of Obstetrics and Gynecology,

The Second Hospital of Jilin University, No. 218 Ziqiang Street, Changchun 130041, Jilin, China

${ }^{2}$ Department of Obstetrics and Gynecology, The Second Hospital of Jilin

University, No. 218 Ziqiang Street, Changchun 130041, Jilin, China
}

commonly characterized by persistent ovulatory disorder, ovarian polycystic morphology, hyperandrogenism, insulin resistance (IR), hyperinsulinemia, and obesity [5, 6]. In addition, in women with PCOS, the risk of type 2 diabetes, cardiovascular disease, infertility, and some adverse endometrial outcomes increases [7-11]. The diversity of the clinical features of PCOS is attributed to the multifactorial contribution on its pathogenesis, including complex genetic and environmental factors [12]. Patients with PCOS often have endometrial abnormalities and most are anovulatory or oligo ovulatory. However, after the anovulation or oligo 
ovulation is treated, they still have lower pregnancy rates and higher spontaneous miscarriage rates, which suggest the decrease of their endometrial receptivity $[10,13]$. Additionally, patients with PCOS have a significantly higher risk of having endometrial hyperplasia and developing endometrial cancer [11]. These adverse endometrial outcomes are associated with the metabolic abnormalities of PCOS including chronic unopposed estrogen, IR, hyperinsulinemia, hyperandrogenism, and obesity, and complex genetic alterations [11, 14]. However, the underlying mechanisms of PCOS in the uterus are still unclear.

Long non-coding RNAs (lncRNAs) are defined as a class of non-coding transcripts with the length of more than 200 nucleotides. Although lncRNAs lack the capacity to code for proteins, they can regulate gene expression at epigenetic, transcriptional, posttranscriptional, and other levels [15]. lncRNAs are proven to play key roles in many biological processes, including genetic imprinting, X-chromosome inactivation, gene transcription regulation, organelle biogenesis, and subcellular trafficking [16]. Dysfunctional lncRNAs contribute to the pathogenesis of many human diseases, such as diabetic nephropathy, nonalcoholic steatohepatitis, cardiomyopathy, atherosclerosis, and cancers in various systems [17-21]. The role of lncRNAs in the pathogenesis of several endometrial diseases has also been reported in recent studies, including implantation failure or spontaneous miscarriage, endometrial hyperplasia, adenomyosis, endometriosis, and endometrial cancer [11, 22-24]. However, we knew little about the role of lncRNAs in the pathogenesis of adverse endometrial outcomes of PCOS.

In our previous research, we found that lncRNA CD36005 (CD36-005) was significantly upregulated in the ovaries of PCOS rat model by lncRNA expression profile analysis [25]. After determining that CD36-005 is also highly expressed in the uteri of PCOS rat model in the present study, we suggest that the upregulation of CD36005 expression might be associated with the pathogenesis of PCOS in the uterus. We used primary endometrial stromal cells from rat uteri as the in-vitro model, and performed RNA sequencing (RNA-seq) technology and bioinformatics analyses after CD36-005 overexpression to investigate its potential role from a more comprehensive perspective. We also conducted CCK- 8 assay to determine the effect of CD36-005 on the proliferation activity of stromal cells, which is the first step of decidualization. Our results provide insights into the underlying molecular mechanisms of CD36-005 in the regulation of endometrial stromal cells and the pathogenesis of adverse endometrial outcomes of PCOS.

\section{Methods}

\section{Animals}

All animal procedures were approved by the Institutional Animal Care and Use Committee of Jilin
University, and were conducted in accordance with the Guidelines for the Care and Use of Laboratory Animals. Mature female Wistar rats (8 weeks old) were purchased for the isolation of primary endometrial stromal cells in the present study.

\section{Animal model, vaginal smears, tissue sampling, and hormone assays}

The uterine tissue used in this study was collected from the PCOS rat model in the article "Expression profiles of mRNA and long noncoding RNA in the ovaries of letrozole-induced polycystic ovary syndrome rat model through deep sequencing [25]." The middle of the uteri (body of uterus) was collected for subsequent total RNA extraction.

\section{Isolation of endometrial stromal cells}

Primary endometrial stromal cells were isolated from 8-week-old Wistar rats' uteri using previously described methods, cultured with DMEM-nutrient mixture F-12 Ham (DMEM-F12, Hyclone, USA) containing 10\% heat-inactivated fetal bovine serum (FBS, Gibco, USA), and incubated at $37^{\circ} \mathrm{C}$ with $5 \% \mathrm{CO}_{2}[26]$.

\section{Cell immunofluorescence}

Stromal cells were seeded on coverslips and washed with phosphate buffer saline (PBS, Hyclone, USA). Then, the confluent cells were fixed in cold methanol, permeabilized with $0.1 \%$ Triton X-100, and blocked with $1 \%$ goat serum. Stromal cells were incubated overnight at $4{ }^{\circ} \mathrm{C}$ with rabbit anti-mouse monoclonal antibody specific to vimentin and cytokeratin-19 at a 1:200 dilution in PBS (Boster, Wuhan, China) [27-29]. In order to validate the specificity of vimentin antibody, $5 \%$ nonimmune goat serum was used as negative control. After washed by PBS three times, stromal cells were incubated with goat-anti-rabbit second antibody (Boster, Wuhan, China) for $2 \mathrm{~h}$ at room temperature. Finally, coverslips were rinsed and mounted with DAPI. The stained stromal cells were observed using Olympus IX71 fluorescence microscope and images were analyzed by using cellSens Dimension.

\section{Overexpression and knockdown of IncRNA CD35-005 in endometrial stromal cells}

When stromal cells reached $70 \%$ confluency, they were transiently transfected with either Ad-CD36-005 or Ad-GFP designed by Hanbio Biotechnology Co., Ltd. (Shanghai, China) at the designated multiplicity of infection (MOI) of 50. The full-length sequence of CD36-005 was directly cloned into the pHBAD-EF1-MCS-3flag-CMV-GFP vector by seamless cloning. After $6 \mathrm{~h}$ incubation at $37^{\circ} \mathrm{C}$ in $5 \% \mathrm{CO} 2$, the medium was replaced with fresh growth 
medium. After transfection with Ad-CD36-005 or Ad-GFP, the stromal cells were collected at $48 \mathrm{~h}$.

The small-interfering RNA (siRNA) duplexes for targeting CD36-005, as well as a scrambled sequence (control siRNA duplex, negative control) were synthesized by the RiboBio Company (Guangzhou, China). The sequences were shown as follows: 5'-UAAGGACCUCUAUUGCUUG TT and CAAGCAAUAGAGGUCCUUATT (CD36-005 siRNA); 5'-UUCUCCGAACGUGUCACGUTT and 5'-A CGUGACACGUUCGGAGAATT (nonspecific scrambled siRNA, negative control). Transfections for siRNA were performed according to Fugene HD Transfection Reagent (Promega, USA) protocol. After transfection with CD36005 or control siRNA, stromal cells were collected at $36 \mathrm{~h}$.

\section{Cell proliferation}

CCK-8 reagent (Promega, USA) was used to perform the proliferation assays according to the manufacturer's directions. Stromal cells were seeded at a density of $1 \times 10^{5}$ /well in 96-well plates and cultured in the DMEM/F12 medium containing 2\% heat-inactivated FBS. After transfection with Ad-CD36-005, Ad-GFP, or CD36-005 siRNA, the stromal cells were cultured for $48 \mathrm{~h}$. Finally, cells in each well were added with $10 \mu \mathrm{l}$ of CCK- 8 reagent and incubated for $2 \mathrm{~h}$. Absorbance was measured at $490 \mathrm{~nm}$ using a 96-well plate reader.

\section{RNA extraction}

Total RNA from the middle of the uteri and stromal cells were extracted using TRIzol (Invitrogen/Life Technologies, USA) according to the manufacturer's protocol. The concentration and quality of RNA were determined using NanoDrop 2000 spectrophotometer (Thermo Fisher Scientific, UK) to ensure that the OD260/280 absorbance ratios of all samples were between 1.8 and 2 . RNA integrity was evaluated using the Agilent 2100 Bioanalyzer (Agilent Technologies, USA).

\section{RNA sequencing and bioinformatics analysis}

RNA samples from three stromal cells in each group with RNA Integrity Number (RIN) $\geq 7$ were subjected to the subsequent mRNA sequencing by Shanghai OE Biotech Co., Ltd. (Shanghai, China). The libraries were constructed using TruSeq Stranded mRNA LTSample Prep Kit (Illumina, San Diego, CA, USA) according to the manufacturer's instructions. These libraries were sequenced on the Illumina sequencing platform (HiSeqTM 2500 or Illumina HiSeq X Ten) and $125 \mathrm{bp} /$ 150 bp paired-end reads were generated. We used Gene Ontology (GO) to categorize the function of the differentially expressed mRNAs and Kyoto Encyclopedia of Genes and Genomes (KEGG) to predict the signaling pathways in which these differentially expressed mRNAs may be involved.

\section{Real-time quantitative PCR analysis}

Reverse transcription reactions were performed using PrimeScript RT Reagent Kit (Takara Bio) according to the manufacturer's protocol. Quantitative real-time polymerase chain reaction (qRT-PCR) analyses were performed at the following conditions: $95{ }^{\circ} \mathrm{C}$ for $2 \mathrm{~min}$ followed by 40 cycles of $95{ }^{\circ} \mathrm{C}$ for $15 \mathrm{~s}$ and $59{ }^{\circ} \mathrm{C}$ for $30 \mathrm{~s}$, according to the instructions of SYBR Premix Ex Taq (TaKaRa). The mRNAs and lncRNA CD36-005 were normalized to glyceraldehyde-3-phosphate dehydrogenase (GAPDH), and the relative expression levels were analyzed by calculating the fold changes using the $2^{-\Delta \Delta \mathrm{Ct}}$ value method. We purchased the primer sequences for qRT-PCR from RiboBio Company (Guangzhou, China).

\section{Statistics}

Significance of difference between two groups was compared by Independent-Samples $\mathrm{T}$ Test. Data are shown mean \pm SEM. Significance of difference was considered significant at $P<0.05$. All statistical analyses were performed using SPSS17.0 software (SPSS Inc., Chicago).

\section{Results \\ Quality control of primary endometrial stromal cell cultures}

The purity of the primary endometrial stromal cells was assessed using the difference in vimentin and cytokeratin expression. The absence of primary antibody was used as a negative control. Results of cell immunofluorescence show that the purity of endometrial stromal cells was more than $90 \%$, which can be used for subsequent experiments (Fig. 1).

Expression of IncRNA CD36-005 in the uteri of PCOS model Result of the qRT-PCR analyses showed that the expression level of CD36-005 in the uteri of PCOS rat model was significantly higher than in the normal (Fig. 2).

\section{Overexpression and knockdown of IncRNA CD36-005 in primary endometrial stromal cells}

Result of the qRT-PCR analyses showed that the expression level of CD36-005 in the Ad-CD36-005 transfected stromal cells was significantly higher compared with that of Ad-GFP transfected cells; CD36-005 expression level in the CD36-005 siRNA transfected stromal cells was significantly lower compared with that of the negative control siRNA transfected cells (Fig. 3).

\section{Effects of IncRNA CD36-005 on primary endometrial stromal cell proliferation}

Overexpression of CD36-005 could strengthen the proliferation activity of stromal cells. On the contrary, the proliferation activity of stromal cells was reduced 

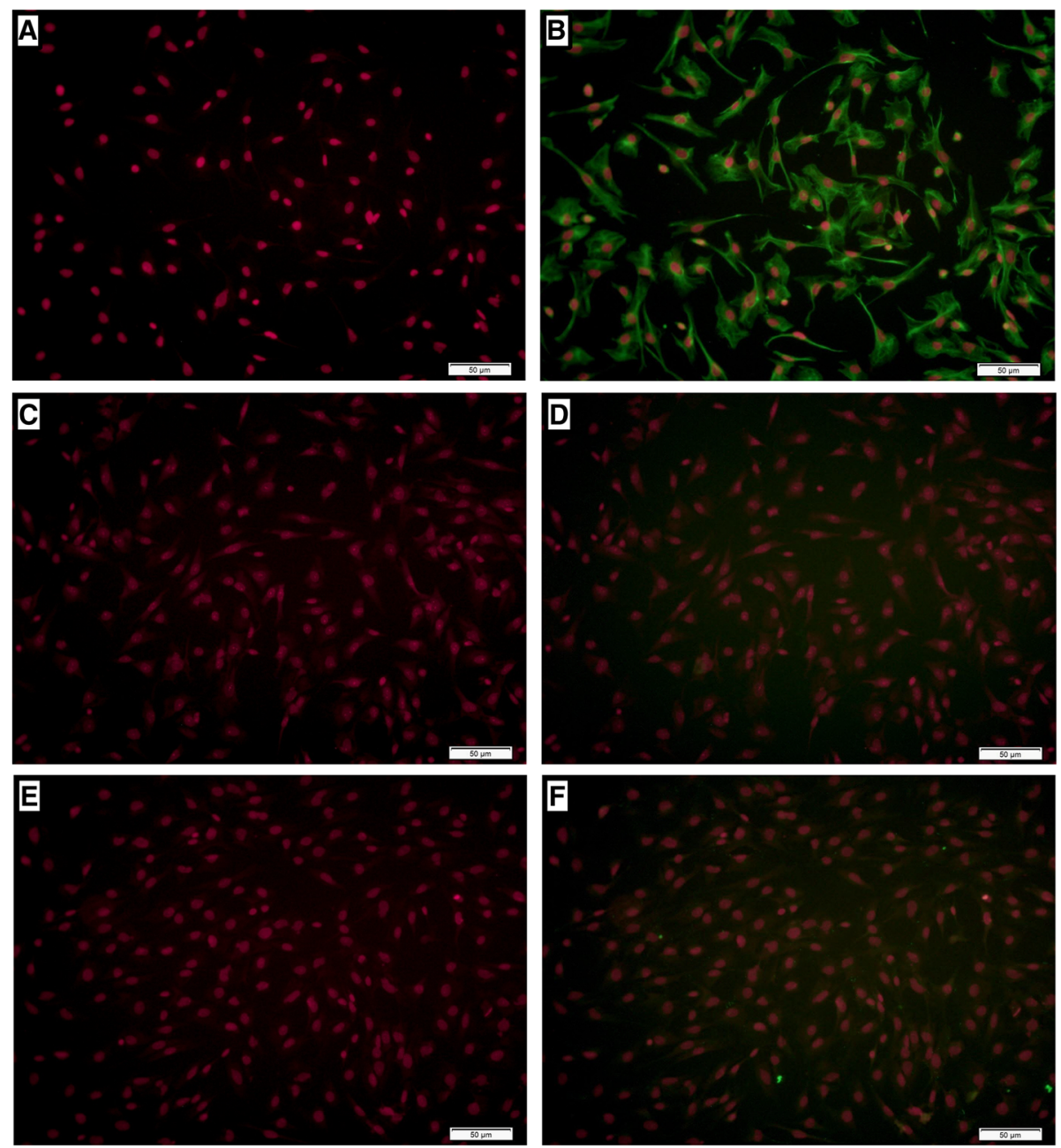

Fig. 1 Immunofluorescence for vimentin in endometrial stromal cells. a, c, e Only DAPI staining on endometrial stromal cell cultures. b Vimentin double staining on endometrial stromal cell cultures. $\mathbf{d}$ Negative control with primary antibody omitted for endometrial stromal cell cultures.

f Cytokeratin-19 double staining on endometrial stromal cell cultures. The scale bar is shown in the lower right corner of each picture. The length of the scale bar is equivalent to $50 \mu \mathrm{m}$

compared with control after they were transfected with CD36-005 siRNA (Fig. 4).

\section{Gene expression profiling following CD36-005 overexpression}

A total of 55 mRNAs were differentially expressed between the Ad-CD36-005 and Ad-GFP groups (absolute $\log _{2}($ fold change) $>1, p<0.05$ ) (Fig. 5). Moreover, 22 mRNAs were differentially expressed with absolute $\log _{2}$ (fold change) $>2$. Among the 55 differentially expressed mRNAs, 28 mRNAs were upregulated and 27 mRNAs were downregulated in the Ad-CD36-005 group.

\section{Validation of differentially expressed mRNAs}

According to references and our interest, six mRNAs from the results of RNA-seq were selected for further validation by using qRT-PCR analysis. Compared with the Ad-GFP group, Hmgn5, Nr5a2, Dll4, Entpd1, and Brms1 displayed a decreased expression, and Fam50a displayed an increased expression in the Ad-CD36-005 group $(\mathrm{p}<0.05)$ (Fig. 6). These results showed that the qRT-PCR results of expression levels of all six mRNAs confirmed were consistent with the RNA-seq data.

\section{Bioinformatics analysis}

Results of the GO analyses categorized the differentially expressed mRNAs into different biological processes, such as biological adhesion, reproductive process, and metabolic process (Fig. 7). KEGG pathway analyses predicted that the differentially expressed mRNAs were involved in various biochemical pathways, including cell growth and death, transport and catabolism, signal transduction, lipid metabolism, and so on (Fig. 8). 

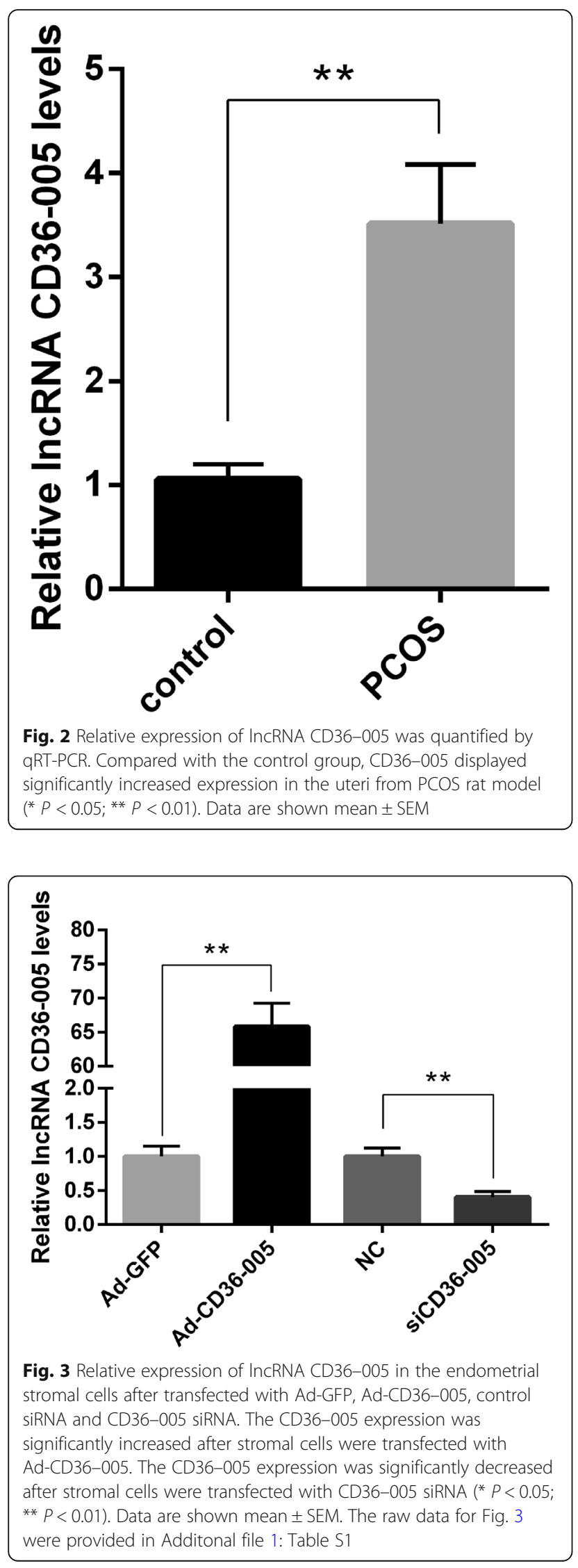

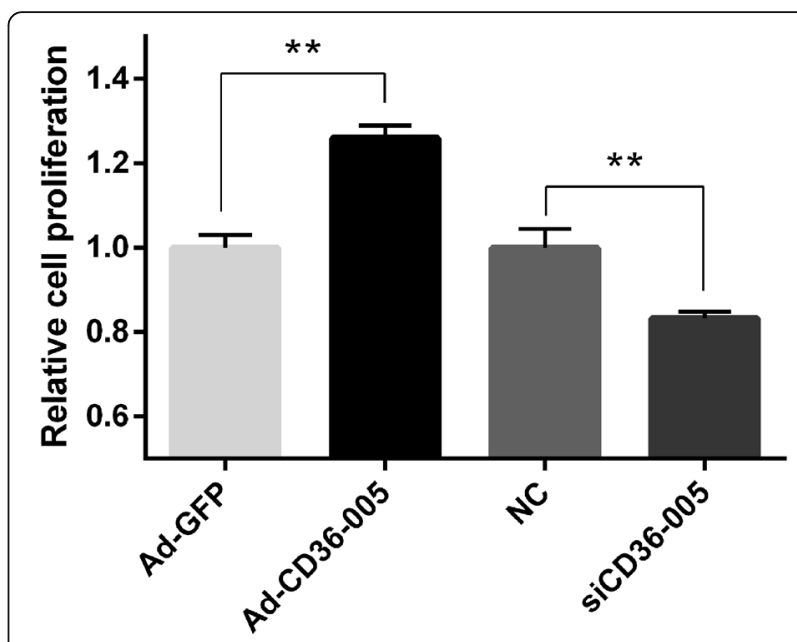

Fig. 4 The effect of IncRNA CD36-005 overexpression and knockdown on the proliferation of endometrial stromal cells. The stromal cells proliferation was significantly increased after stromal cells were transfected with Ad-CD36-005. The stromal cells proliferation was decreased after stromal cells were transfected with CD36-005 siRNA ( $P<0.05 ;{ }^{* *} P<0.01$ ). Data are shown mean \pm SEM. The raw data for Fig. 4 were provided in Additonal file 2: Table $\$ 2$

\section{Discussion}

Accumulating evidence shows that the endocrinal and metabolic disorders of PCOS have complex effects on the endometrium, leading to endometrial abnormalities $[11,14]$. The endometrium is a steroid hormone-targeting tissue that undergoes cyclic secretion and proliferation dynamically in response to estrogen and progesterone produced by the ovaries [30]. Because most patients with PCOS are anovulatory or oligo ovulatory, the endometrium is continuously stimulated by unopposed estrogen in the absence of the regulatory effects of progesterone, which decrease the endometrial receptivity and promote the development of endometrial hyperplasia and even cancer in the long run [11]. In addition, patients with PCOS often have IR and obesity [6]. Insulin levels in the local endometrium affect the endometrial development and receptivity. IR can cause hyperglycemia, which further aggravates hyperandrogenism [14]. These are all high risk factors of endometrial cancer [11]. The pathogenesis of PCOS and its abnormal endometrial outcomes is a multifactorial biological process that involves a large number of genes and biological pathways, among which the role of lncRNAs has been studied in recent years [22, 31].

Owing to the abundance but low expression level of lncRNAs, they were initially considered to be transcriptional noise without any biological function [32]. With the rapid development of genomics and transcriptomics technology, researchers gradually found the regulatory 


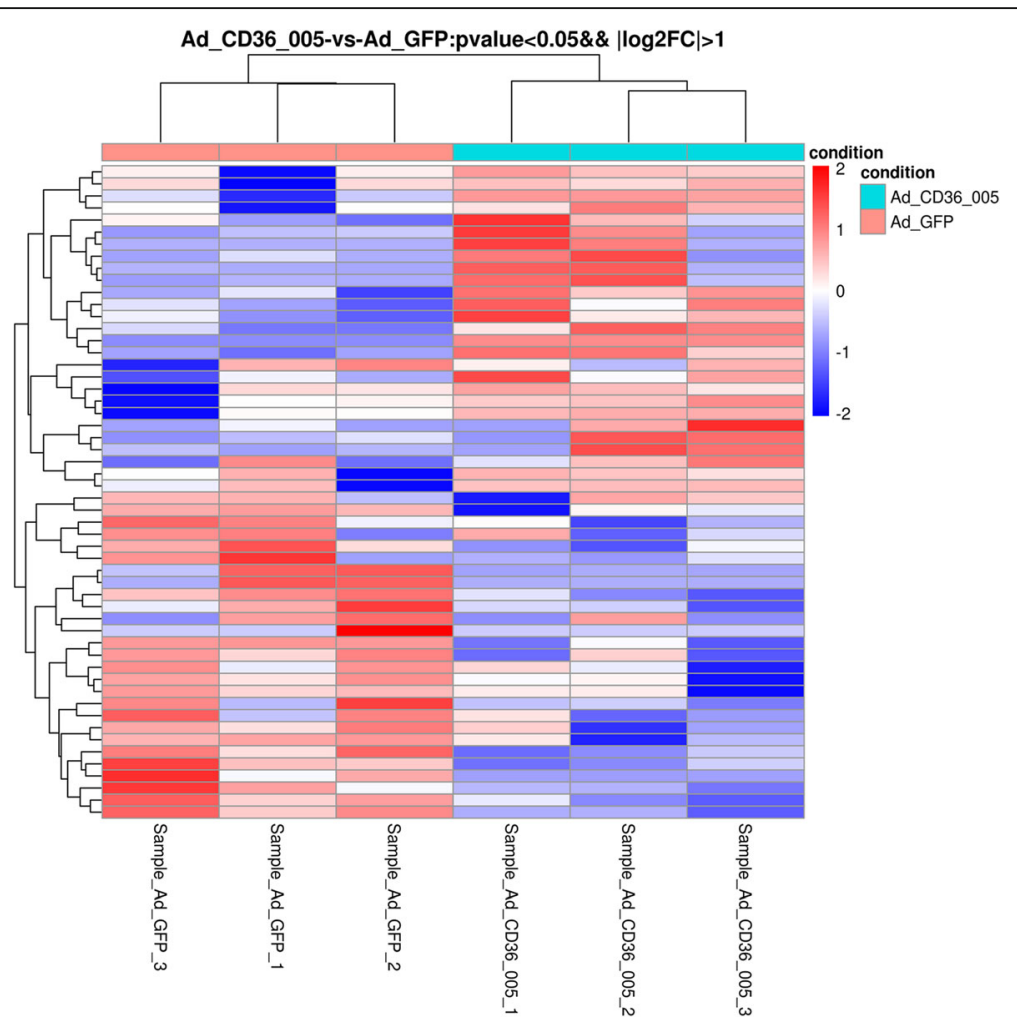

Fig. 5 Hierarchical clustering heatmaps of differentially expressed mRNAs in the endometrial stromal cells after IncRNA CD36-005 overexpression

role of lncRNAs in various human diseases [17]. In accumulating studies, only a few lncRNAs have been reported to be associated with both PCOS and various endometrial disorders. The expression of steroid receptor RNA activator (SRA) as well as lncRNA CTBP1-AS, a novel androgen receptor modulator, was significantly higher in peripheral blood leukocytes of women with
PCOS. Meantime, it is known that women with PCOS show dysregulated hormone receptors expression, suggesting us a potential role of genes modulating hormone receptors in PCOS-associated endometrial disorders [33-35]. However, the functions and underlying mechanisms of these dysregulated remains unclear and need to be further studied.

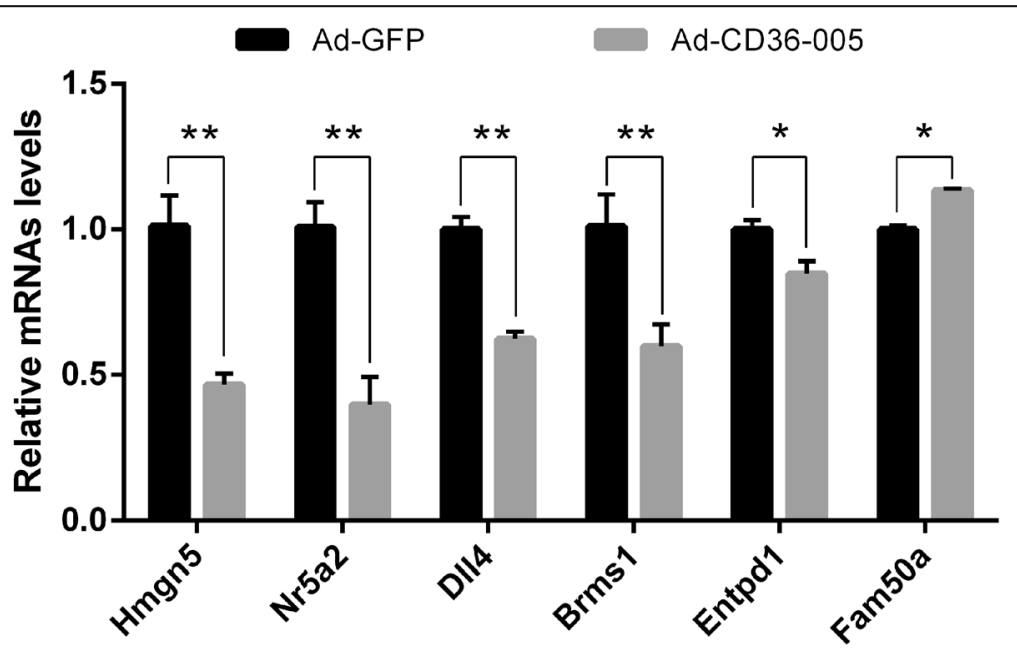

Fig. 6 Relative expression of six selected mRNA was quantified by qRT-PCR. Compared with the Ad-GFP group, Hmgn5, Nr5a2, D\|l4, Entpd1, and Brms1 displayed decreased expression, whereas Fam50a displayed an increased expression in the Ad-CD36-005 group $\left({ }^{*} P<0.05 ;{ }^{* *} P<0.01\right)$. Data are shown mean \pm SEM 


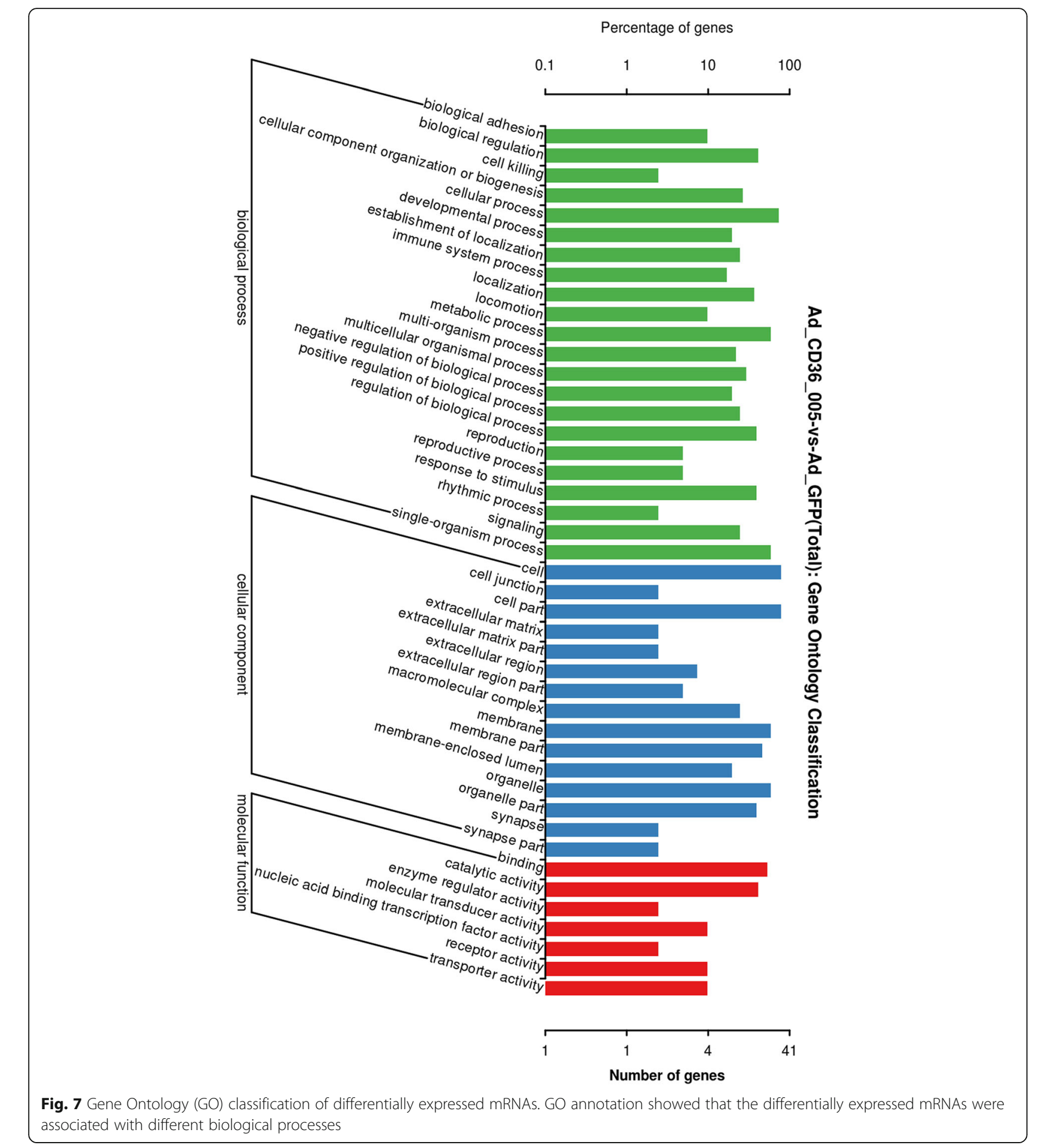

The endometrial stromal cell is one of the in-vitro models of the endometrial tissue used for the study of the molecular mechanisms of endometrial diseases. Decidualization is a process of endometrial stromal cell proliferation and subsequent differentiation, during which stromal cells transform into specialized decidual cells [36, 37]. This process is essential for embryo implantation and successful pregnancy. Although many studies have focused on the molecular mechanisms of decidualization, known lncRNAs associated with decidualization were limited. HK2P1 is a lncRNA found to be decreased in the decidua of severe preeclampsia patients. In vitro results show that downregulated HK2P1 inhibited human endometrial stromal cell (HESC) proliferation and 


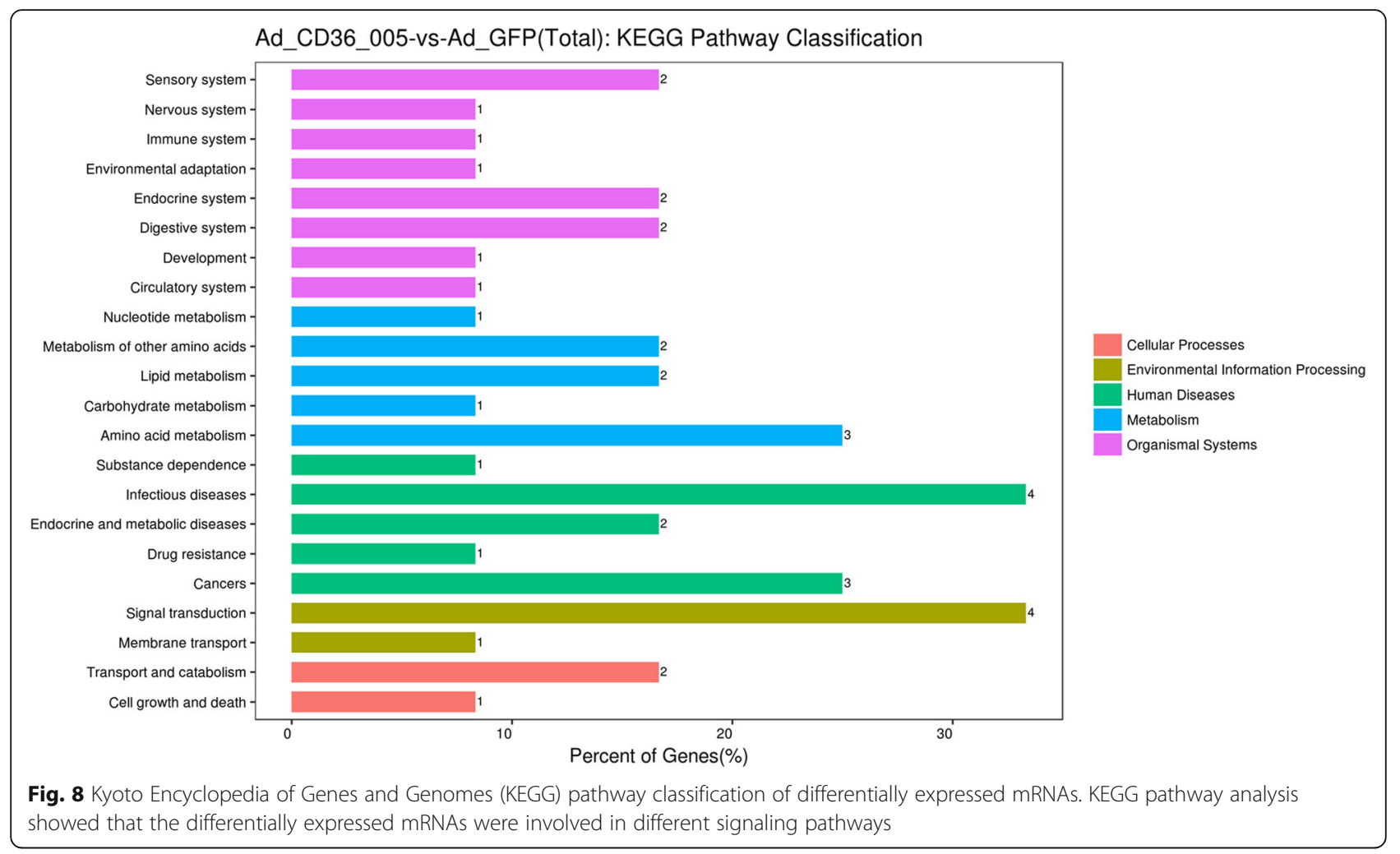

differentiation by regulating miR-6887-3p and its target gene HK2 expression as a ceRNA [38]. The expression of another lncRNA, LINC00473, is highly induced in HESCs after decidual stimulus [39]. These studies prove a crucial role of lncRNA in stromal cell decidualization and suggest that IncRNAs participate in some endometrial diseases partly through affecting stromal cells, of which some may be caused by PCOS.

In the present study, CD36-005 was upregulated in the uteri of PCOS rat model and could promote the proliferation of stromal cells. However, the molecular mechanisms remain to be characterized. Thus, we conducted RNA-seq and found 55 known mRNAs in the primary endometrial stromal cells differentially expressed between the Ad-CD36-005 and Ad-GFP group with a threshold of $p<$ 0.05 and $\mid \log _{2}$ (Fold-Change) $\mid>1$. We chose six mRNAs (Hmgn5, Nr5a2, Dll4, Entpd1, Fam50a, and Brms1) for qRT-PCR validation, and the results were consistent with the RNA-seq data. In previous studies, Qiao performed microarray analysis on the endometrial biopsies of women with PCOS during the implantation window and found down-regulated genes were associated with endometrial receptivity [40]. Similarly, Bellver performed microarray hybridization and identified an aberrant endometrial transcriptome in obese women with PCOS during the implantation window [41]. Among our RNA-seq result, several genes have been reported to be involved in endometrial disorders, but little is known whether these endometrial disorders are related to PCOS. Hmgn5, also known as Nsbp1, regulates uterine decidualization as a downstream gene of Hoxa10 in a differentiation-specific manner [42]. Hmgn5 was also found to be hypomethylated in mouse uteri when exposed to diethylstilbestrol or fenistein neonatally [43]. Nr5a2, also known as Lrh-1, was known as a key transcriptional factor of multiple steroidogenic genes in vitro [44]. In Wang's study, $\mathrm{Nr} 5 \mathrm{a} 2$ promotes aromatase expression in primary rat granulosa cells, indicating its potential involvement in PCOS while the ovaries of women with PCOS have abnormal steroidogenesis and folliculogenesis [45, 46]. Consistent with above report, Kevin elucidated in his review that a higher level of Lrh-1 could activate estradiol production in the endometrium of women with endometrial cancer [47]. Lrh-1 is also essential for a successful pregnancy as its indispensible roles in the luteal function, decidualization, and placental formation [48]. Combined with our result, we could speculate that the dysregulated expression of Nr5a2 may be associated with PCOS-associated endometrial disorders. Dll4, a gene involved in the delta-notch pathways, participates in the decidualization failure of stromal cells from women with endometriosis [49]. The promiscuous expression of Dll4 impaired decidual angiogenesis, and coordinated with disrupted decidual cellular proliferation and apoptosis, could be one of the causes of early miscarriages [50]. No relevant studies focus on the 
relationship of Entpd1, Fam50a, and Brms1 to endometrial disorders, and further studies are needed.

GO and KEGG analyses were performed to identify the potential functions and pathways of the 55 target genes of CD36-005. GO analyses revealed that these differentially expressed genes were involved in various categories, such as nitric oxide biosynthetic process and positive regulation of gene expression including toll-like receptor 7/9 (TLR-7/ 9) and interleukin-6/8 (IL-6/8). The nitric oxide (NO)/ nitric oxide synthase (NOS) system was presumed to locally regulate the endometrial functions, including stromal cells decidualization [51]. Nos2, a synthase involved in NO production, was upregulated by CD36-005 overexpression and predicted to be involved in the nitric oxide mediated signal transduction, nitric-oxide synthase activity, and nitric-oxide synthase binding. Nos 2 was also found to participate in the inflammatory process of PCOS and some endometrial abnormalities [52, 53]. Thus, we can speculate that Nos2 may play a role in the pathogenesis of endometrial abnormalities of PCOS, however, there is no direct evidence and further studies are needed. TLR-7/9 and IL-6/8 were shown to be related to endometrial disorders. The expression of TLR-9 was increased with reduced DNA methylation in spontaneous preterm labor [54]. Higher TLR-9 transcriptional activity may be a protective factor for endometrial cancer risk [55]. According to Gu, the expression of TLR9 in cumulus cells was influenced significantly by PCOS, which may further lower the embryo quality and decrease the fertility rate of women with PCOS. These results suggest us the dysregulated expression of TLR9 may be potentially involved in the pathogenesis of PCOS and its adverse endometrial outcomes. TRL-7 is crucial in the establishment and maintenance of pregnancy in sheep [56]. IL-8 participates in the pathogenesis of endometriosis by regulating ectopic endometrial cell proliferation, invasion, and adhesion [57]. Levels of IL-6 in the mid-secretory-phase endometrium are lower in women with previous recurrent miscarriage [58]. Additionally, the aberrant IL- 6 and IL- 8 in endometrial stromal fibroblasts from women with PCOS was thought be related to the altered endometrial immune profile and imbalanced leukocyte migration, both of which contribute to a sub-optimal implantation of women with PCOS [59]. Thus, the regulation of these biological processes may be one of the complex mechanisms of some endometrial abnormalities, of which some might be induced by PCOS.

KEGG analyses showed that these differentially expressed genes were associated with diverse signaling pathways, including cell growth and death, transport and catabolism, signal transduction, lipid metabolism, endocrine and metabolic diseases, and cancers. Among these pathways, lipid metabolism was a biological process closely related to PCOS and its endometrial abnormalities. In previous studies, $70 \%$ of women with PCOS were found to have dyslipidemia, which is also a risk factor for cardiovascular disease and endometrial cancer [60]. Patients with PCOS commonly suffer from increased body mass index, total cholesterol, triglyceride, low-density lipoprotein-cholesterol, and decreased high-density lipoprotein-cholesterol [61]. In Chekir's study, patients with PCOS have an increased uterine arterial pulsatility index and reduced endometrial thickness during the luteal phase, which may cause reproductive failure. The endometrial dysfunction induced by impaired uterine perfusion was thought to be correlated with dyslipidemia [61]. Nieman regarded endometrial cancer as the most relevant cancer with obesity. Adipocytes secrete adipokines and mediate the transition of androgens to estrogen, which promotes the development of endometrial cancer [62]. Mogat1 is a gene upregulated by CD36-005 overexpression and predicted to be involved in lipid metabolism. Hence, the differential upregulation of Mogat1 may participate in the pathogenesis of PCOS and its endometrial abnormalities through lipid metabolism. Above all, these results suggest a potential mechanism through which the dysregulated mRNAs involved in different categories and biological pathways contribute together to the regulation of stromal cells and the pathogenesis of endometrial abnormalities, some of which may be caused by PCOS.

\section{Conclusion}

We identified a list of 55 potential target mRNAs of CD36-005 in the primary endometrial stromal cells by RNA-seq and confirmed the relative expression levels of Hmgn5, Nr5a2, Dll4, Entpd1, Fam50a, and Brms1 using qRT-PCR. We used GO and KEGG pathway analyses, and predicted the various biochemical pathways that regulate the proliferation and decidualization of stromal cells and the pathogenesis of some endometrial abnormalities, in which these differentially expressed mRNAs may be involved in. Our results partly explained the mechanisms by which CD36-005 regulate the etiology and pathophysiology of PCOS in the uterus of rat with letrozole-induced PCOS, and laid a foundation for further studies on the molecular function and mechanism of CD36-005 in stromal cells. Further detailed studies are needed to clarify the underlying mechanisms of CD36-005 on the regulation of stromal cells and to investigate if these alterations could serve as biomarkers for the prediction of some endometrial diseases induced by PCOS.

\section{Additional files}

Additional file 1: Table S1. The raw data for Figure 3. (XLSX $9 \mathrm{~kb}$ ) Additional file 2: Table S2. The raw data for Figure 4. (XLSX 9 kb) 


\section{Abbreviations}

CD36-005: IncRNA CD36-005; E2: 17-beta estradiol; GO: Gene Ontology; HESC: human endometrial stromal cell; IL: interleukin; IR: insulin resistance; KEGG: Kyoto Encyclopedia of Genes and Genomes; LncRNA: long non-coding RNA; MOl: multiplicity of infection; NO: nitric oxide; NOS: nitric oxide synthase; PCOS: polycystic ovary syndrome; RNA-seq: RNA sequencing; SRA: steroid receptor RNA activator; TLR: toll-like receptor

\section{Availability of data and materials}

The datasets used and/or analysed during the current study are available from the corresponding author on reasonable request.

\section{Authors' contributions}

$X Z, L Z$ and $M C$ designed the study; $X Z, Y X, L F$ and DL performed the experiments; XZ wrote the manuscript. All authors discussed the results, read and approved the final manuscript.

\section{Ethics approval and consent to participate}

The animal protocols were carried out according to the Guide for the Administration of Laboratory Animals (Directive 86/609/EEC on the Protection of Animals Used for Experimental and Other Scientific Purposes, 1986) and ratified by the Institutional Animal Care and Use Committee (IACUC) of Jilin University.

\section{Consent for publication}

Not applicable.

\section{Competing interests}

The authors declare that they have no competing interests.

\section{Publisher's Note}

Springer Nature remains neutral with regard to jurisdictional claims in published maps and institutional affiliations.

Received: 10 July 2018 Accepted: 25 September 2018 Published online: 15 October 2018

\section{References}

1. Li R, Zhang Q, Yang D, Li S, Lu S, Wu X, et al. Prevalence of polycystic ovary syndrome in women in China: a large community-based study. Hum Reprod. 2013;28:2562-9.

2. Asuncion M, Calvo RM, San Millan JL, Sancho J, Avila S, Escobar-Morreale HF. A prospective study of the prevalence of the polycystic ovary syndrome in unselected Caucasian women from Spain. J Clin Endocrinol Metab. 2000; 85:2434-8.

3. March WA, Moore VM, Willson KJ, Phillips DI, Norman RJ, Davies MJ. The prevalence of polycystic ovary syndrome in a community sample assessed under contrasting diagnostic criteria. Hum Reprod. 2010;25:544-51.

4. Azziz R, Woods KS, Reyna R, Key TJ, Knochenhauer ES, Yildiz BO. The prevalence and features of the polycystic ovary syndrome in an unselected population. J Clin Endocrinol Metab. 2004;89:2745-9.

5. Christakou C, Diamanti-Kandarakis E. Polycystic ovary syndrome-phenotypes and diagnosis. Scand J Clin Lab Invest Suppl. 2014;244:18-22 discussion 1.

6. Ranasinha S, Joham AE, Norman RJ, Shaw JE, Zoungas S, Boyle J, et al. The association between polycystic ovary syndrome (PCOS) and metabolic syndrome: a statistical modelling approach. Clin Endocrinol. 2015;83:879-87.

7. Kakoly NS, Khomami MB, Joham AE, Cooray SD, Misso ML, Norman RJ, et al. Ethnicity, obesity and the prevalence of impaired glucose tolerance and type 2 diabetes in PCOS: a systematic review and meta-regression. Hum Reprod Update. 2018;24:455-67.

8. Bilal M, Haseeb A, Rehman A. Relationship of polycystic ovarian syndrome with cardiovascular risk factors. Diabetes Metab Syndr. 2018:12:375-80.

9. Joham AE, Teede HJ, Ranasinha S, Zoungas S, Boyle J. Prevalence of infertility and use of fertility treatment in women with polycystic ovary syndrome: data from a large community-based cohort study. J Women's Health (2002). 2015;24:299-307

10. Shang K, Jia X, Qiao J, Kang J, Guan Y. Endometrial abnormality in women with polycystic ovary syndrome. Reprod Sci. 2012;19:674-83.

11. Giudice LC. Endometrium in PCOS: implantation and predisposition to endocrine CA. Best Pract Res Clin Endocrinol Metab. 2006;20:235-44.
12. Franks S, McCarthy Ml, Hardy K. Development of polycystic ovary syndrome: involvement of genetic and environmental factors. Int J Androl. 2006:29: 278-85 discussion 86-90.

13. Bahri Khomami M, Boyle JA, Tay CT, Vanky E, Teede HJ, Joham AE, et al. Polycystic ovary syndrome and adverse pregnancy outcomes: current state of knowledge, challenges and potential implications for practice. Clin Endocrinol. 2018;88:761-9.

14. Jeanes YM, Reeves S. Metabolic consequences of obesity and insulin resistance in polycystic ovary syndrome: diagnostic and methodological challenges. Nutr Res Rev. 2017;30:97-105.

15. An S, Song JJ. The coded functions of noncoding RNAs for gene regulation. Mol Cells. 2011;31:491-6.

16. Guttman M, Amit I, Garber M, French C, Lin MF, Feldser D, et al. Chromatin signature reveals over a thousand highly conserved large non-coding RNAs in mammals. Nature. 2009:458:223-7.

17. Taft RJ, Pang KC, Mercer TR, Dinger M, Mattick JS. Non-coding RNAs: regulators of disease. J Pathol. 2010;220:126-39.

18. Alvarez ML, Distefano JK. The role of non-coding RNAs in diabetic nephropathy: potential applications as biomarkers for disease development and progression. Diabetes Res Clin Pract. 2013;99:1-11.

19. Sookoian S, Flichman D, Garaycoechea ME, San Martino J, Castano GO, Pirola CJ. Metastasis-associated lung adenocarcinoma transcript 1 as a common molecular driver in the pathogenesis of nonalcoholic steatohepatitis and chronic immune-mediated liver damage. Hepatol Commun. 2018;2:654-65.

20. Yang W, Li Y, He F, Wu H. Microarray profiling of long non-coding RNA (IncRNA) associated with hypertrophic cardiomyopathy. BMC Cardiovasc Disord. 2015;15:62

21. Wu G, Cai J, Han Y, Chen J, Huang ZP, Chen C, et al. LincRNA-p21 regulates neointima formation, vascular smooth muscle cell proliferation, apoptosis, and atherosclerosis by enhancing p53 activity. Circulation. 2014;130:1452-65.

22. Panir K, Schjenken JE, Robertson SA, Hull ML. Non-coding RNAs in endometriosis: a narrative review. Hum Reprod Update. 2018;24:497-515.

23. Chen MY, Liao GD, Zhou B, Kang LN, He YM, Li SW. Genome-wide profiling of long noncoding RNA expression patterns in women with repeated implantation failure by RNA sequencing. Reprod Sci. 2018: 1933719118756752. https://doi.org/10.1177/1933719118756752.

24. Wang $\mathrm{H}_{\text {, }}$ Cao $\mathrm{O}$, Ge J, Liu C, Ma Y, Meng Y, et al. LncRNA-regulated infection and inflammation pathways associated with pregnancy loss: genome wide differential expression of IncRNAs in early spontaneous abortion. Am J Reprod Immunol. 2014;72:359-75.

25. Fu LL, Xu Y, Li DD, Dai XW, Xu X, Zhang JS, et al. Expression profiles of mRNA and long noncoding RNA in the ovaries of letrozole-induced polycystic ovary syndrome rat model through deep sequencing. Gene. 2018;657:19-29.

26. Tian XC, Wang QY, Li DD, Wang ST, Yang ZQ, Guo B, et al. Differential expression and regulation of Cryab in mouse uterus during preimplantation period. Reproduction. 2013;145:577-85.

27. Yuhki M, Kajitani T, Mizuno T, Aoki Y, Maruyama T. Establishment of an immortalized human endometrial stromal cell line with functional responses to ovarian stimuli. Reprod Biol Endocrinol. 2011;9:104.

28. Shen M, Liu X, Zhang H, Guo SW. Transforming growth factor beta signaling coincides with epithelial-mesenchymal transition and fibroblast-tomyofibroblast transdifferentiation in the development of adenomyosis in mice. Hum Reprod. 2016;31:355-69.

29. De Clercq K, Hennes A, Vriens J. Isolation of mouse endometrial epithelial and stromal cells for in vitro Decidualization. J Vis Exp. 2017. https://doi.org/ $10.3791 / 55168$

30. Gellersen B, Brosens JJ. Cyclic decidualization of the human endometrium in reproductive health and failure. Endocr Rev. 2014;35:851-905.

31. Takenaka K, Chen BJ, Modesitt SC, Byrne FL, Hoehn KL, Janitz M. The emerging role of long non-coding RNAs in endometrial cancer. Cancer Genet. 2016:209:445-55.

32. Struhl K. Transcriptional noise and the fidelity of initiation by RNA polymerase II. Nat Struct Mol Biol. 2007:14:103-5.

33. Liu Z, Hao C, Huang X, Zhang N, Bao H, Qu Q. Peripheral blood leukocyte expression level of IncRNA steroid receptor RNA activator (SRA) and its association with polycystic ovary syndrome: a case control study. Gynecol Endocrinol. 2015:31:363-8.

34. Liu Z, Hao C, Song D, Zhang N, Bao H, Qu Q. Androgen receptor Coregulator CTBP1-AS is associated with polycystic ovary syndrome in Chinese women: a preliminary study. Reprod Sci. 2015;22:829-37. 
35. Piltonen TT. Polycystic ovary syndrome: endometrial markers. Best Pract Res Clin Obstet Gynaecol. 2016;37:66-79.

36. Dey SK, Lim H, Das SK, Reese J, Paria BC, Daikoku T, et al. Molecular cues to implantation. Endocr Rev. 2004:25:341-73.

37. Zhang $\mathrm{S}$, Lin $\mathrm{H}$, Kong $\mathrm{S}$, Wang $\mathrm{S}$, Wang $\mathrm{H}$, Wang $\mathrm{H}$, et al. Physiological and molecular determinants of embryo implantation. Mol Asp Med. 2013;34:939-80.

38. Lv H, Tong J, Yang J, Lv S, Li WP, Zhang C, et al. Dysregulated Pseudogene HK2P1 May Contribute to Preeclampsia as a Competing Endogenous RNA for Hexokinase 2 by Impairing Decidualization. Hypertension. 2018;71:648-58.

39. Liang XH, Deng WB, Liu YF, Liang YX, Fan ZM, Gu XW, et al. Non-coding RNA LINC00473 mediates decidualization of human endometrial stromal cells in response to CAMP signaling. Sci Rep. 2016;6:22744.

40. Qiao J, Wang L, Li R, Zhang X. Microarray evaluation of endometrial receptivity in Chinese women with polycystic ovary syndrome. Reprod BioMed Online. 2008;17:425-35.

41. Bellver J, Martinez-Conejero JA, Labarta E, Alama P, Melo MA, Remohi J, et al. Endometrial gene expression in the window of implantation is altered in obese women especially in association with polycystic ovary syndrome. Fertil Steril. 2011;95:2335-41 41.e1-8.

42. Li DD, Zhao SY, Yang ZQ, Duan CC, Guo CH, Zhang HL, et al. Hmgn5 functions downstream of Hoxa10 to regulate uterine decidualization in mice. Cell Cycle. 2016;15:2792-805.

43. Tang WY, Morey LM, Cheung YY, Birch L, Prins GS, Ho SM. Neonatal exposure to estradiol/bisphenol a alters promoter methylation and expression of Nsbp1 and Hpcal1 genes and transcriptional programs of Dnmt $3 a / b$ and $M b d 2 / 4$ in the rat prostate gland throughout life. Endocrinology. 2012;153:42-55.

44. Saxena D, Escamilla-Hernandez R, Little-Ihrig L, Zeleznik AJ. Liver receptor homolog-1 and steroidogenic factor-1 have similar actions on rat granulosa cell steroidogenesis. Endocrinology. 2007;148:726-34.

45. Wang Q, Kim JY, Xue K, Liu JY, Leader A, Tsang BK. Chemerin, a novel regulator of follicular steroidogenesis and its potential involvement in polycystic ovarian syndrome. Endocrinology. 2012;153:5600-11.

46. Garg D, Merhi Z. Relationship between advanced glycation end products and steroidogenesis in PCOS. Reprod Biol Endocrinol. 2016;14:71.

47. Mouzat K, Baron S, Marceau G, Caira F, Sapin V, Volle DH, et al. Emerging roles for LXRs and LRH-1 in female reproduction. Mol Cell Endocrinol. 2013; 368:47-58.

48. Zhang C, Large MJ, Duggavathi R, DeMayo FJ, Lydon JP, Schoonjans K, et al. Liver receptor homolog-1 is essential for pregnancy. Nat Med. 2013;19: 1061-6.

49. Su RW, Strug MR, Joshi NR, Jeong JW, Miele L, Lessey BA, et al. Decreased notch pathway signaling in the endometrium of women with endometriosis impairs decidualization. J Clin Endocrinol Metab. 2015;100: E433-42.

50. Garcia-Pascual CM, Ferrero H, Zimmermann RC, Simon C, Pellicer A, Gomez R. Inhibition of Delta-like 4 mediated signaling induces abortion in mice due to deregulation of decidual angiogenesis. Placenta. 2014;35:501-8.

51. Yoshiki N, Kubota T, Matsumoto Y, Aso T. Expression of inducible nitric oxide synthase in human cultured endometrial stromal cells. Mol Hum Reprod. 1999;5:353-7.

52. Wang XR, Hao HG, Chu L. Glycyrrhizin inhibits LPS-induced inflammatory mediator production in endometrial epithelial cells. Microb Pathog. 2017; 109:110-3.

53. Schmidt J, Weijdegard B, Mikkelsen AL, Lindenberg S, Nilsson L, Brannstrom M. Differential expression of inflammation-related genes in the ovarian stroma and granulosa cells of PCOS women. Mol Hum Reprod. 2014;20:49-58.

54. Walsh SW, Chumble AA, Washington SL, Archer KJ, Sahingur SE, Strauss JF 3rd. Increased expression of toll-like receptors 2 and 9 is associated with reduced DNA methylation in spontaneous preterm labor. J Reprod Immunol. 2017;121:35-41.

55. Ashton KA, Proietto A, Otton G, Symonds I, McEvoy M, Attia J, et al. Toll-like receptor (TLR) and nucleosome-binding oligomerization domain (NOD) gene polymorphisms and endometrial cancer risk. BMC Cancer. 2010;10:382

56. Ruiz-Gonzalez I, Minten M, Wang X, Dunlap KA, Bazer FW. Involvement of TLR7 and TLR8 in conceptus development and establishment of pregnancy in sheep. Reproduction. 2015;149:305-16.

57. Sikora J, Smycz-Kubanska M, Mielczarek-Palacz A, Kondera-Anasz Z. Abnormal peritoneal regulation of chemokine activation-the role of IL-8 in pathogenesis of endometriosis. Am J Reprod Immunol. 2017;77. https://doi. org/10.1111/aji.12622.
58. Jasper MJ, Tremellen KP, Robertson SA. Reduced expression of IL-6 and IL1alpha mRNAs in secretory phase endometrium of women with recurrent miscarriage. J Reprod Immunol. 2007;73:74-84.

59. Piltonen TT, Chen JC, Khatun M, Kangasniemi M, Liakka A, Spitzer T, et al. Endometrial stromal fibroblasts from women with polycystic ovary syndrome have impaired progesterone-mediated decidualization, aberrant cytokine profiles and promote enhanced immune cell migration in vitro. Hum Reprod. 2015;30:1203-15.

60. Legro RS, Kunselman AR, Dunaif A. Prevalence and predictors of dyslipidemia in women with polycystic ovary syndrome. Am J Med. 2001; 111:607-13.

61. Chekir C, Nakatsuka M, Kamada Y, Noguchi S, Sasaki A, Hiramatsu Y. Impaired uterine perfusion associated with metabolic disorders in women with polycystic ovary syndrome. Acta Obstet Gynecol Scand. 2005;84:189-95.

62. Nieman KM, Romero IL, Van Houten B, Lengyel E. Adipose tissue and adipocytes support tumorigenesis and metastasis. Biochim Biophys Acta 2013;1831:1533-1541.

\section{Ready to submit your research? Choose BMC and benefit from:}

- fast, convenient online submission

- thorough peer review by experienced researchers in your field

- rapid publication on acceptance

- support for research data, including large and complex data types

- gold Open Access which fosters wider collaboration and increased citations

- maximum visibility for your research: over $100 \mathrm{M}$ website views per year

At BMC, research is always in progress.

Learn more biomedcentral.com/submissions 